\title{
Quality Assurance in Bone Densitometry
}

\author{
Giuseppe Guglielmi • Danila Diano • \\ Federico Ponti · Alberto Bazzocchi
}

Published online: 20 December 2013

(c) Springer Science+Business Media New York 2013

\begin{abstract}
Dual energy X-ray absorptiometry (DXA) is a well-established method for the diagnosis of osteoporosis and monitoring of treatment. Recent advances in DXA technologies have improved accuracy and precision. Nevertheless, errors in bone mineral density measurement and interpretation could occur and negatively affect the diagnosis and management of patients. These errors can result from improper equipment installation and maintenance, inadequate education and training in bone densitometry, and insufficient knowledge of current clinical guidelines. This review describes the current procedures of quality assurance in bone densitometry and emphasizes strict adherence of technicians and clinicians to manufacturers' recommendations and current clinical guidelines.
\end{abstract}

Keywords Osteoporosis - Absorptiometry, photon · Quality control · Quality assurance · Health care . Radiation dosage $\cdot$ Radiation protection

This article is part of the Topical Collection on Osteoporosis Imaging.

\section{G. Guglielmi ( ()}

Department of Radiology, University of Foggia, Viale Luigi

Pinto 1, 71100 Foggia, Italy

e-mail: giuseppe.guglielmi@unifg.it

G. Guglielmi

Department of Radiology, Scientific Institute "Casa Sollievo della Sofferenza” Hospital, Viale Cappuccini 1,

71013 San Giovanni Rotondo, Foggia, Italy

D. Diano $\cdot$ F. Ponti

Department of Specialized, Diagnostic, and Experimetal Medicine, Sant'Orsola-Malpighi Hospital, University of Bologna, Via G. Massarenti 9, 40138 Bologna, Italy

\section{A. Bazzocchi}

Diagnostic and Interventional Radiology, "Rizzoli" Orthopaedic Institute, Via G.C. Pupilli 1, 40136 Bologna, Italy

\section{Introduction}

Osteoporosis is the most common bone disease and represents a major public health problem because of its worldwide increasing incidence and heavy social economic burden [1].

Currently, it is estimated that over 200 million people worldwide suffer from this disease, and at least $40 \%$ of women and about $20 \%$ of men will sustain one or more fragility fractures in their lifetime [2].

Osteoporosis causes more than 8.9 million fractures annually worldwide, and over one-third of all osteoporotic fractures occur in Europe [3, 4]. By 2050, the worldwide number of hip fractures is estimated to rise from 1.66 to 6.26 million [5], and their incidence is projected to increase up to $240 \%$ in women and $310 \%$ in men [4].

Osteoporotic fractures cause increasing morbidity and mortality rates; in particular, hip fractures result in $10-20 \%$ of mortality within 1 year $[1,6]$.

Osteoporosis has been defined on the basis of bone mineral density (BMD) assessed at the hip or lumbar spine level. BMD represents a cornerstone for the general management of osteoporosis, being used for diagnosis, fracture risk assessment, selection of patients for treatment and monitoring of treatment efficacy [7].

According to the World Health Organization (WHO) criteria, osteoporosis is defined as a BMD value less than or equal to 2.5 standard deviations below the young normal mean reference population.

Dual energy X-ray absorptiometry (DXA) is the current gold standard for BMD measurement.

BMD measured by DXA is highly correlated with biomechanical bone strength and, in longitudinal studies, with fracture risk [7]. 
High-quality BMD measurement and interpretation are important because physicians rely on these reports to make patient care decisions, including additional medical evaluations (laboratory or imaging), starting or changing drug therapy, or suggesting some dietetic and lifestyle habits [8].

Incorrect $\mathrm{BMD}$ acquisition or reporting may generate unnecessary medical expenses and lead to inappropriate clinical and therapeutic decisions that could have adverse effects on patients' health [9]. Errors can result from improper equipment installation and maintenance, inadequate education and training in bone densitometry and insufficient knowledge of current guidelines.

BMD testing, interpretation and reporting are erroneously considered to be simple; instead, all these steps require training and experience for technologists and physicians.

For these reasons, mistakes in BMD assessment are commonly seen. In 2006, Lewiecki et al. [8] reported about $71 \%$ of clinicians and $45 \%$ of technologist see incorrect densitometric interpretation and acquisition at least once a month. These errors were due to a lack of adherence to manufacturers' recommendations for device maintenance and quality control, incorrect acquisition and analysis of data (e.g., wrong demographic information, improper patient positioning, artifacts not removed from the scanned area, incorrect labeling of vertebral bodies), or misleading interpretation of results.

Hence, performing the periodic and daily machine calibration tests conforming to the manufacturers' guidelines, an adequate acquisition and analysis of data, and correct interpretation and reporting of results are essential steps to help the management of patients $[8,10]$.

The aim of this review is to discuss the current practice of quality assurance (QA) procedures in bone densitometry.

\section{Quality Assurance}

QA is by definition "a programme for the systematic monitoring and assessment of the various aspects of a service or facility to ensure that standards of quality are being met." QA in bone densitometry has to guarantee (1) the maintainance of adequate equipment performance with the lowest possible radiation dose to patients consistent with clinical imaging requirements and (2) the assurance of adequate diagnostic information provided at the lowest possible cost. A QA program should include (1) guidelines and requirements for equipment installation and its clinical use, (2) standardised periodic tests developed to maintain diagnostic quality (quality control, QC), (3) preventive maintenance procedures, (4) staff training and education, and (5) radiation safety policies and procedures.

\section{Equipment and Installation}

Manufacturers should provide all necessary and required equipment for DXA facilities (e.g., the positioning device for the anteroposterior lumbar spine scan acquisition; the hip positioning device for the femur scan acquisition).

The supplier of the equipment should describe all clinical applications of the system and specify the performance in terms of accuracy and precision [11••]. Equipment packages should also include dedicated phantoms for calibration and QC procedures.

The installation of the equipment should be followed by procedures that guarantee correct functioning and satisfy all current radiation protection requirements and manufacturer specifications [12•].

\section{Diagnostic Quality and Quality Control: Phantom Scanning and Cross-Calibration}

When performing DXA measurements, it is important to implement a QA program to guarantee that any observed density changes are real and not due to machine or to operator variability. The quality of the acquired data is defined by accuracy and precision [13].

Accuracy is the ability of a measurement to match the actual value of the quantity being measured and is expressed in terms of accuracy error. The accuracy error is the percentage difference between a measurement and a defined standard and is influenced by the machine. Generally, the accuracy error of the most widely used bone densitometers is about 5-10\% [14].

To evaluate the accuracy of a DXA, a daily calibration measurement, according to the manufacturer's instructions, is performed using a standard phantom supplied with the system. In addition to the daily calibration, the manufacturers recommand a periodic QC (generally every 3-6 months) using a different phantom to independently monitor the scanner stability [13]. The various manufacturers have developed different types of QC phantoms. Several of these consist of vertebrae made of simulated bone material encased in plastic; others are made of an aluminum bar to be submersed in water. During the calibration process, the phantom has to be located in the middle of the scanning table, at the top side of the table. At the end of the calibration, results have to be analyzed and recorded in the QC database [13].

Precision is defined as the reproducibility and is expressed in terms of precision error. Precision error provides a quantitative measure of the ability of densitometric systems to detect changes in BMD over time and thus is an important component of clinical decision making. This is influenced by several parameters, such as the skill and 
training of the technician performing the scan, the skeletal site of measurement and the clinical features of patients [12•].

Knowledge of the precision error is essential in clinical practice to guarantee a correct diagnosis and management of patients, because it allows distinguishing between true change in BMD and random variability in the measurement procedure. The least significant change (LSC) enables the identification of statistically significant changes in BMD by considering the precision error [coefficient of variation $(\mathrm{CV})]$ of the measurement [15•]. LSC should to be calculated using the International Society for Clinical Densitometry (ISCD) precision calculating tool at www.iscd.org.

The various densitometer manufacturers often establish precision errors for their equipment on the order of $1 \%$ [16]. Since the precision errors estimated by the manufacturer do not usually represent the error in the clinical setting, the ISCD recommends that in vivo precision errors and LSC for each DXA center should be determined using repeated measurements in 30 subjects (representative of the clinic's patient population) with repositioning or in 15 subjects scanned three times [17, 18••].

Moreover, if a DXA facility has more than one technologist, every technologist should perform an in vivo precision assessment using patients. Then an average precision error combining data from all technologists should be used to establish precision error and consequently LSC for the facility.

The ISCD also recommends that each technologist should complete precision assessment after having performed approximately 100 patient scans [18••].

The LSC of the most commonly used DXA machines are reported in Table 1 [15•].

Differences in calibration exist among the densitometry systems from different manufacturers. To overcome this relevant limit, cross-calibration techniques have been developed. In the last 20 years cross-calibration was attempted using a European Spine Phantom containing three simulated vertebrae of different densities (low, normal and high density) composed of calcium hydroxyapatite. Results were compared to the spine BMD of a group of females and then were used to define and introduce the

Table 1 Precision of bone measurement expressed in terms of least significant change (LSC) using the most common DXA technologies $[15 \bullet]$

\begin{tabular}{llll}
\hline & $\begin{array}{l}\text { L1-L4 } \\
\text { BMD }(\%)\end{array}$ & $\begin{array}{l}\text { Total hip } \\
\text { BMD }(\%)\end{array}$ & $\begin{array}{l}\text { Femoral neck } \\
\text { BMD }(\%)\end{array}$ \\
\hline Lunar expert & 1.0 & 0.9 & 1.5 \\
Lunar prodigy & 1.0 & 1.1 & 1.5 \\
Hologic & 1.0 & 1.2 & 1.6 \\
Lunar iDXA & 0.4 & 0.6 & 1.3 \\
\hline
\end{tabular}

standardized BMD (sBMD). sBMDs were used to correct the calibration differences among the three major distributors of DXA (Hologic, Lunar and Norland) [19, 20]. The ISCD guidelines actually affirm that is not possible to quantitatively compare BMD or to calculate a LSC between different facilities without cross-calibration.

The ISCD also recommends performing a cross-calibration when the DXA hardware, but not the entire system, have been changed, or when a system with the same technology (manufacturer and model) has been replaced, in order to allow a quantitative comparison to the prior machine. The cross-calibration procedure consists of ten phantom scans, with repositioning, before and after hardware change [18••]. If a greater than $1 \%$ difference in mean BMD is observed, the manufacturer should be contacted for service or correction. When changing an entire system to one made by the same manufacturer using a different technology, or when changing to a system made by a different manufacturer, cross-calibration should be performed in 30 patients representative of the facility's patient population once on the initial system and then twice on the new system within 60 days. All cross-calibration measures have to be performed at the hip and lumbar site. The average BMD and LSC between the initial and new machine should be calculated using the ISCD DXA Machine Cross-Calibration Tool [18••]. Then LSC for comparison between the previous and new system should be used. Inter-system quantitative comparisons can only be made if cross-calibration is performed on each commonly measured skeletal site. Once a new precision assessment has been performed on the new system, all future scans should be compared to scans performed on the new system using the newly established intra-system LSC. If a crosscalibration assessment is not performed, no quantitative comparison to the prior machine can be made. Consequently, a new baseline BMD and intra-system LSC need to be established [18••].

\section{Preventive Maintenance Procedures}

In addition to QC, a periodic assessment of the condition of the DXA devices should be executed (i.e., checking the electrical safety, equipment emergency stop, radiation warning lights, and the availability and adequacy of patient support tools). Moreover, all test results should be recorded to detect needed changes [12•].

\section{Staff Training and Education}

Incorrect acquisition and analysis of data or incorrect interpretations of results occur frequently in daily clinical 
Table 2 Effective dose of different DXA devices

\begin{tabular}{|c|c|c|c|c|c|}
\hline & Technology & L1-L4 ( $\mu \mathrm{Sv})$ & Hip $(\mu \mathrm{Sv})$ & Wrist $(\mu \mathrm{Sv})$ & LVA $(\mu \mathrm{Sv})$ \\
\hline GE-Lunar DPX & Pencil-beam & 0.21 & 0.15 & & \\
\hline Hologic QDR1000 & Pencil beam & $0.50-2.60$ & $0.90-1.80$ & 0.07 & \\
\hline GE-Lunar expert & Fan beam (wide-angle) & 59.00 & $50.00-56.00$ & & 38.00 \\
\hline Hologic discovery & Fan beam (wide-angle) & $4.40-13.30$ & $3.10-9.30$ & & $10.00-41.00$ \\
\hline GE-Lunar Prodigy & Fan beam (narrow-angle) & $0.20-1.60$ & $0.15-1.20$ & 0.01 & 2.50 \\
\hline GE-Lunar iDXA & Fan beam (narrow-angle) & 3.37 & 3.09 & & $8.00-12.09$ \\
\hline
\end{tabular}

practice [8] and are responsible for misleading diagnoses and inadequate patient management.

Therefore, training of technicians performing the examination as well as education of radiologists writing the reports is needed to prevent and minimize these kinds of errors.

Errors in performing DXA acquisitions and data analysis are usually due to (1) improper patient positioning (e.g., the spine is not parallel to the edges of the DXA table or the hip is not sufficiently internally rotated); (2) using the wrong scan mode (e.g., the scan mode may alter the BMD and is manually or automatically selected); (3) artifacts not being removed from the scanned area (e.g., the spine is scanned when the patient is wearing an underwire bra or has a bellybutton ring); (4) obtaining incorrect demographic information (e.g., a man is entered as a woman or the incorrect age is used); (5) failure to correct improper default identification of bone edges and regions of interest (e.g., the computer includes large osteophyte or vascular calcifications in the area of the measured spine); (6) incorrect labeling of vertebral bodies [9, 21].

Errors in the interpretations of BMD values also frequently occur and are due to (1) an incorrect application of WHO diagnostic T-score criteria and ISCD official positions (e.g., reporting T-scores in a healthy premenopausal woman and applying the WHO diagnostic criteria); (2) an invalid BMD comparison (LSC not known, different instruments used, left hip compared with right hip, comparing T-scores instead of BMD, different scan modes); (3) fracture risk incorrectly represented [9].

Moreover, body composition may influence the precision and accuracy of DXA and therefore affect interpretations of DXA examinations. In particular, caution must be used when interpreting BMD results in subjects who have undergone large weight changes [22].

However, training courses for both physicians and technologists are provided by several national and international societies.

\section{Dose Radiation Exposure}

One of the main strengths of DXA is the low radiation exposure for patients $[10,23]$. Nevertheless, recent advances in DXA technology (e.g., spatial resolution improvement, small time consumption) have involved an increase in radiation dose [24]. However, radiation exposure is much lower than other diagnostic techniques and lower than the average daily dose from natural background radiation (6.6 $\mu \mathrm{Sv} /$ day; $2,400 \mu \mathrm{Sv} /$ year) $[12 \bullet, 24]$.

Many variables affect the DXA patient dose, such as the site of measurement (e.g., lumbar spine, forearm, hip), patient body size, acquisition technique, X-ray tube filtration, efficiency of detection system, number of scans, exposure parameters, scan speed and scan size [11••].

Table 2 shows the effective dose (calculated using the organ- or tissue-absorbed doses and the relative radiosensitivity assigned to each of these organs or tissues [25]) of several models of DXA.

Pregnancy is a contraindication to DXA. Damilakis et al. [26] estimated conceptus doses and risks related to DXA performed during pregnancy. A careful screening for pregnancy is essential to minimize the number of accidental exposures of pregnant patients by DXA. When a pregnant woman has a DXA examination, communication with her about the dose to the fetus is very important. Because the risks from DXA are negligible, usually abortion due to radiation exposure is not recommended $[25,26]$.

The radiation burden is increased in follow-up studies. A clinical justification of these examinations should be provided to ensure that the expected benefits exceed the potential risks [11••].

The occupational radiation dose should also be considered. The radiation exposure for DXA technologists is generally very low if all procedures are performed according to the manufacturer's guidelines. The reported scatter dose rates at $1 \mathrm{~m}$ from the central axis of the patient table range from a few tenths of $1-5 \mu \mathrm{Sv} / \mathrm{h}$ depending on the scanner model. From these values, recent calculations and measurements indicate that the annual dose from fan-beam systems for an average workload (20 patients/day) at $1 \mathrm{~m}$ from the scanner will be between 0.1 and $1.5 \mathrm{mSv}$, depending on the scanner model [10, 27].

If a female technician becomes pregnant, she should inform her employer. A pregnant staff member can continue working in a DXA scanning room as long as the 
fetal dose is kept below $1 \mathrm{mGy}$ for the term of the pregnancy [28].

An increase in the operator's distance from the patient and the use of mobile or immobile shielding can reduce the operator's radiation exposure [10]. The need for shielding mainly depends on the patient workload, size of the examination room and type of DXA scanner (especially for fan-beam technology) [12•].

Fan-beam and cone-beam systems may require shielding if the operator has to be seated close to the table or if the walls are close to the table [27].

Moreover, if the room configuration does not allow the necessary distance between the DXA and operator, a transparent lead-acrylic shielding may be needed to protect the technicians during the examination [10].

\section{Conclusions}

QA procedures in bone densitometry should be performed carefully and strictly conform to current guidelines to guarantee adequate diagnostic performance and a consequent correct overview and management of osteoporotic patients.

\section{Compliance with Ethics Guidelines}

Conflict of Interest Giuseppe Guglielmi, Danila Diano, Federico Ponti, and Alberto Bazzocchi declare that they have no conflicts of interest.

Human and Animal Rights and Informed Consent This article does not contain any studies with human or animal subjects performed by the authors.

\section{References}

Papers of particular interest, published recently, have been highlighted as:

- Of importance,

-• Of major importance

1. Cauley JA. Public health impact of osteoporosis. J Gerontol A. 2013;68:1243-51.

2. World Health Organization. Assessment of osteoporosis at the primary health care level. Summary Report of a WHO Scientific Group. www.who.int/chp/topics/rheumatic/en/index.html. Accessed 2007.

3. Johnell O, Kanis JA. An estimate of the worldwide prevalence and disability associated with osteoporotic fractures. Osteoporos Int. 2006;17:1726-33.

4. Gullberg B, Johnell O, Kanis JA. World-wide projections for hip fracture. Osteoporos Int. 1997;7:407-13.
5. International Osteoporosis Foundation. http://www.iofbonehealth. org/epidemiology. Accessed 2006.

6. Cummings SR, Melton LJ. Epidemiology and outcomes of osteoporotic fractures. Lancet. 2002;359:1761-7.

7. Link TM. Osteoporosis imaging: state of the art and advanced imaging. Radiology. 2012;263:3-17.

8. Lewiecki EM, Binkley N, Petak SM. DXA quality matters. J Clin Densitom. 2006;9:388-92.

9. Lewiecki EM, Lane NE. Common mistakes in the clinical use of bone mineral density testing. Nat Clin Pract Rheumatol. 2008;4: 667-74.

10. Larkin A, Sheahan N, O'Connor U, Gray L, Dowling A, Vano E, Torbica P, Salat D, Schreiner A, Neofotistou V, et al. QA/ acceptance testing of DEXA X-ray systems used in bone mineral densitometry. Radiat Prot Dosim. 2008;129:279-83.

11. • Guglielmi G, Damilakis J, Solomou G, Bazzocchi A. Quality assurance of imaging techniques used in the clinical management of osteoporosis. Radiol Med 2012;117:1347-54. This review is about the quality assurance procedures for densitometric and imaging techniques commonly used to diagnose and manage osteoporosis.

12. - Damilakis J, Adams JE, Guglielmi G, Link TM. Radiation exposure in X-ray-based imaging techniques used in osteoporosis. Eur Radiol. 2010;20:2707-14. This study provides information about the radiation exposure and radiation safety associated with the most commonly used X-ray-based imaging techniques in osteopososis.

13. Faulkner KG. Clinical use of bone densitometry. In: Marcus R, Feldman D, Kelsey J, editors. Osteoporosis, Two-Volume Set. 2nd ed. San Diego: Academic Press; 2001 chapter 59.

14. Blake GM, Fogelman I. How important are BMD accuracy errors for the clinical interpretation of DXA scans? J Bone Miner Res. 2008;23:457-62.

15. • Hind K, Oldroyd B, Truscott JG. In vivo precision of the GE Lunar iDXA densitometer for the measurement of total-body, lumbar spine, and femoral bone mineral density in adults. J Clin Densitom. 2010;13:413-7. This study provides the precision for bone measurements acquired using the most recent DXA technology (Lunar iDXA) and the previous ones (Lunar Expert, Lunar Prodigy and Hologic).

16. Baim S, Wilson CR, Lewiecki EM, Luckey MM, Downs RW Jr. Lentle BC: precision assessment and radiation safety for dualenergy X-ray absorptiometry: position paper of the International Society for Clinical Densitometry. J Clin Densitom. 2005;8: 371-8.

17. Leslie WD, Moayyeri A. Minimum sample size requirements for bone density precision assessment produce inconsistency in clinical monitoring. Osteoporos Int. 2006;17:1673-80.

18. •• International Society for Clinical Densitometry: 2013 ISCD Official Positions. http://www.iscd.org/official-positions/2013iscd-official-positions-adult/. Accessed 2013. The new official positions of the ISCD as updated in 2013 about phantom scanning, precision assessment and cross-calibration of DXA machines.

19. Steiger P. Standardization of measurements for assessing BMD by DXA. Calcif Tissue Int. 1995;57:469.

20. Hanson J. Standardization of femur BMD. J Bone Miner Res. 1997;12:1316-7.

21. Libber J, Binkley N, Krueger D. Clinical observations in total body DXA: technical aspects of positioning and analysis. J Clin Densitom. 2012;15:282-9.

22. Yu EW, Thomas BJ, Brown JK, Finkelstein JS. Simulated increases in body fat and errors in bone mineral density measurements by DXA and QCT. J Bone Miner Res. 2012;27: 119-24. 
23. Blake GM, Naeem M, Boutros M. Comparison of effective dose to children and adults from dual X-ray absorptiometry examinations. Bone. 2006;38:935-42.

24. Toombs RJ, Ducher G, Shepherd JA, De Souza MJ. The impact of recent technological advances on the trueness and precision of DXA to assess body composition. Obesity (Silver Spring). 2012;20:30-9.

25. The 2007 Recommendations of the International Commission on Radiological Protection. ICRP publication 103. Ann ICRP. 2007;37:1-332.
26. Damilakis J, Perisinakis K, Vrahoriti H, Kontakis G, Varveris H, Gourtsoyiannis N. Embryo/fetus radiation dose and risk from dual X-ray absorptiometry examinations. Osteoporos Int. 2002;13:716-22.

27. Sheahan NF, Dowling A, O'Reilly G, Malone JF. Commissioning and quality assurance protocol for dual energy X-ray absorptiometry (DEXA) systems. Radiat Prot Dosim. 2005;117:288-90.

28. Radiation Protection of Patients. Staff Radiation Protection DXA. https://rpop.iaea.org/RPOP/RPoP/Content/index.htm. Accessed 2001. 